\title{
Main controlling factors and models of organic matter accumulation in Lower Carboniferous Dawuba
}

\section{formation shale in Southern Guizhou , China}

Jialiang Niu ${ }^{\text {a,b }}$, Jinchuan Zhang a,b, ${ }^{*}$, Xuan Tang a,b,f*, Kun Yuan ${ }^{\text {a,e }}$, Tuo Lin ${ }^{\text {e }}$, Yang Liu ${ }^{\text {a,b }}$, Yanjie Niu ${ }^{\text {d }}$, Pei Li ${ }^{\text {a,c }}$, Xingqi Li a,b, Yutao Liang a,b

a School of Energy Resources, China University of Geosciences, Beijing, 100083, China

${ }^{\mathrm{b}}$ Key Laboratory of Strategy Evaluation for Shale Gas of Ministry of Land and Resources, China University of Geosciences, Beijing, 100083, China

${ }^{\mathrm{c}}$ Petroleum Exploration and Production Research Institute, China Petroleum and Chemical Corporation (SINOPEC), No. 31 Xueyuan Road, Beijing 100083, China;

${ }^{d}$ Gansu coalfield geology bureau 145th team exploration engineering company, Team 145, Zhanghuo Road, Ganzhou District, Zhangye City 734000, China;

e Oil and Gas Survey Center, China Geological Survey, Beijing 100083, China

${ }^{\mathrm{f}}$ State Key Laboratory of Shale Oil and Gas Enrichment Mechanisms and Effective Development

Corresponding Authors

Jinchuan Zhang - China University of Geosciences, Beijing

100083, China;

Email: zhangic@,cugb.edu.cn

Xuan Tang - China University of Geosciences, Beijing

100083, China;

Email: tangxuan@cugb.edu.cn 
Table S1 Major and trace element contents in ZY-1Well samples

\begin{tabular}{|c|c|c|c|c|c|c|c|c|c|c|c|c|c|c|c|c|c|c|c|c|}
\hline Sample No & Depth & Lithology & $\mathrm{Al} \%$ & $\mathrm{Ti} \%$ & $\mathrm{Fe} \%$ & $\mathrm{Mn} \%$ & $\mathrm{~K} \%$ & $\mathrm{Ca} \%$ & $\mathrm{Na} \%$ & $\mathrm{Ba} \%$ & Р\% & $\mathrm{Vppm}$ & Cr ppm & Co ppm & Ni ppm & $\mathrm{Cu} \mathrm{ppm}$ & $\mathrm{Zn} \mathrm{ppm}$ & U ppm & Mo ppm & $\mathrm{Rb} p p m$ \\
\hline ZY1-YX-1 & 2668 & Gray black mudstone & 15.71 & 0.66 & 6.23 & 0.03 & 3.16 & 10.53 & 0.33 & 0.08 & 0.08 & 146.07 & 193.54 & 12.79 & 80.41 & 20.42 & 95.62 & 3.354 & 2.29 & 137.71 \\
\hline ZY1-YX-3 & 2688 & Dark gray mudstone & 15.57 & 0.66 & 5.6 & 0.03 & 3.2 & 11.21 & 0.19 & 0.07 & 0.05 & 133.83 & 180.59 & 10.36 & 67.75 & 17.47 & 60.48 & 2.971 & 1.73 & 131.02 \\
\hline ZY1-4-05 & 2699 & Gray black mudstone & 20.94 & 0.88 & 5.81 & 0.02 & 4.28 & 4.39 & 0.12 & 0.05 & 0.06 & 184.42 & 127.97 & 14.83 & 89.37 & 19.07 & 102.52 & 3.555 & 1.66 & 191.6 \\
\hline ZY1-YX-5 & 2702 & Gray black mudstone & 15.94 & 0.79 & 6.26 & 0.03 & 2.21 & 9.89 & 0.9 & 0.64 & 0.09 & 122.39 & 170.41 & 13.06 & 61.11 & 16.74 & 77.57 & 3.404 & 1.4 & 104.28 \\
\hline ZY1-YX-76 & 2709 & Gray black mudstone & 16.75 & 0.67 & 6.14 & 0.03 & 3.54 & 9.76 & 0.33 & 0.17 & 0.11 & 152.88 & 189.84 & 12.22 & 72.03 & 18.85 & 81.53 & 3.432 & 1.64 & 151.77 \\
\hline ZY1-YX-85 & 2719 & Gray black mudstone & 15.07 & 0.61 & 5.89 & 0.03 & 3.17 & 12.23 & 0.27 & 0.04 & 0.06 & 138.55 & 136.49 & 13.23 & 85.05 & 19.85 & 64.27 & 3.196 & 0.81 & 144.5 \\
\hline ZY1-YX-8 & 2728 & Gray black mudstone & 15.56 & 0.79 & 6.32 & 0.04 & 2.1 & 10.15 & 0.94 & 0.61 & 0.1 & 100.56 & 141.61 & 8.83 & 48.6 & 11.15 & 60.82 & 2.3 & 0 & 51.56 \\
\hline ZY1-YX-9 & 2738 & Grey black shale & 12.71 & 0.64 & 4.81 & 0.02 & 1.76 & 14.01 & 0.78 & 1.04 & 0.18 & 90.71 & 90.1 & 9.58 & 55.53 & 12.11 & 66.61 & 2.287 & 0.55 & 44.29 \\
\hline ZY1-YX-11 & 2750 & Gray black mudstone & 15.48 & 0.64 & 5.56 & 0.02 & 3.68 & 10.7 & 0.14 & 0.32 & 0.07 & 140.04 & 120.84 & 10.71 & 63.69 & 18.69 & 89.99 & 3.719 & 1.19 & 145.06 \\
\hline ZY1-YX-119 & 2759 & Gray black mudstone & 14.12 & 0.64 & 7.48 & 0.04 & 2.32 & 8.77 & 0.73 & 0.93 & 0.15 & 128.09 & 121.41 & 13.99 & 72.27 & 18.14 & 142.53 & 2.915 & 0.75 & 90.6 \\
\hline ZY1-YX-16 & 2770 & Dark gray argillaceous limestone & 7.34 & 0.31 & 3.71 & 0.04 & 1.02 & 31.62 & 0.12 & 0.07 & 0.07 & 78.93 & 63.75 & 6.97 & 53.58 & 10.43 & 48.43 & 2.564 & 0.61 & 35.33 \\
\hline ZY1-YX-136 & 2787 & Gray black mudstone & 14.6 & 0.71 & 5.74 & 0.02 & 1.92 & 3.45 & 0.88 & 0.99 & 0.21 & 126.23 & 169.13 & 10.63 & 48.43 & 15.48 & 95.73 & 3.273 & 1.81 & 98.83 \\
\hline ZY1-6-01 & 2796 & Grey black shale & 15.79 & 0.78 & 5.05 & 0.01 & 2.06 & 1.64 & 2.47 & 0.04 & 0.04 & 37.9 & 65.2 & 7.1 & 25.3 & 3.9 & 0.8 & 0 & 1.91 & 2.3 \\
\hline ZY1-6-03 & 2798.2 & Grey black shale & 18.09 & 0.79 & 6.77 & 0.01 & 2.54 & 1.37 & 1.04 & 0.05 & 0.05 & 53.5 & 91.64 & 10.6 & 38.3 & 6.4 & 1.3 & 0 & 2.77 & 3.7 \\
\hline ZY1-6-05 & 2800.5 & Grey black shale & 20.75 & 0.98 & 5.38 & 0.01 & 2.65 & 0.48 & 1.13 & 0.05 & 0.04 & 46.7 & 77.26 & 8.8 & 31.3 & 5 & 0.9 & 0 & 2.5 & 2.9 \\
\hline ZY1-6-07 & 2802.6 & Grey black shale & 16.66 & 0.89 & 6.25 & 0.01 & 2.01 & 1.97 & 0.92 & 0.04 & 0.22 & 40.9 & 78.53 & 9.2 & 34.4 & 6.9 & 1.4 & 0 & 2.7 & 4.5 \\
\hline ZY1-6-09 & 2804.7 & Grey black shale & 19.3 & 0.87 & 7.22 & 0.01 & 2.54 & 1.31 & 1.04 & 0.06 & 0.08 & 49.3 & 81.37 & 9.6 & 35 & 6.1 & 1.2 & 0 & 2.46 & 3.7 \\
\hline ZY1-7-02 & 2807.55 & Grey black shale & 18.98 & 0.79 & 6.16 & 0.01 & 2.24 & 3.14 & 0.95 & 0.06 & 0.08 & 48.1 & 77.34 & 9.3 & 33.9 & 5.9 & 1.2 & 0 & 2.53 & 3.6 \\
\hline ZY1-7-04 & 2808.5 & Grey black shale & 12.82 & 0.79 & 4.71 & 0.01 & 1.75 & 2.02 & 0.79 & 0.04 & 0.12 & 43.9 & 77.9 & 9.5 & 37.1 & 8.5 & 1.7 & 0 & 2.81 & 5.4 \\
\hline ZY1-7-06 & 2810.5 & Grey black shale & 20.24 & 0.88 & 6.51 & 0.01 & 2.78 & 0.64 & 0.94 & 0.06 & 0.06 & 47.2 & 80.76 & 9.4 & 34.9 & 6.2 & 1.2 & 0 & 2.55 & 3.7 \\
\hline ZY1-7-08 & 2812.5 & Grey black shale & 16.1 & 0.91 & 5.57 & 0.01 & 1.85 & 0.37 & 1.02 & 0.04 & 0.1 & 38.7 & 70.57 & 7.9 & 28.8 & 5.1 & 1 & 0 & 2.31 & 3.1 \\
\hline ZY1-7-10 & 2814.5 & Grey black shale & 18.23 & 0.85 & 6.26 & 0.01 & 2.42 & 0.87 & 1.03 & 0.05 & 0.1 & 44.8 & 71.31 & 8.6 & 31.9 & 5.4 & 1.1 & 0 & 2.57 & 3.5 \\
\hline ZY1-YX-151 & 2823 & Gray black mudstone & 17.72 & 0.84 & 5.35 & 0.01 & 2.31 & 2.96 & 1.4 & 0.15 & 0.08 & 189.88 & 202.66 & 10.36 & 54.64 & 16.67 & 69.75 & 4.128 & 2.5 & 119.15 \\
\hline ZY1-YX-25 & 2828 & Gray black mudstone & 10.98 & 0.53 & 5.8 & 0.04 & 1.68 & 9.38 & 0.59 & 0.38 & 0.08 & 100.56 & 141.61 & 8.83 & 48.6 & 11.15 & 60.82 & 2.3 & 0 & 51.56 \\
\hline ZY1-YX-160 & 2834 & Gray black mudstone & 16.68 & 0.8 & 5.06 & 0.01 & 2.24 & 4.19 & 2.18 & 1.32 & 0.07 & 213.03 & 216.75 & 12.01 & 70.72 & 33.27 & 147.24 & 5.171 & 6.07 & 122.83 \\
\hline ZY1-YX-26 & 2838 & Gray black mudstone & 10.25 & 0.49 & 6.37 & 0.04 & 1.52 & 9.87 & 0.61 & 0.32 & 0.09 & 90.71 & 90.1 & 9.58 & 55.53 & 12.11 & 66.61 & 2.287 & 0.55 & 44.29 \\
\hline ZY1-YX-172 & 2847 & Grey dolomite & 15.42 & 0.72 & 5.15 & 0.02 & 2.11 & 8.28 & 1.9 & 0.46 & 0.1 & 167.04 & 189.88 & 9.58 & 60.27 & 18.01 & 116.89 & 4.309 & 6.88 & 102.21 \\
\hline
\end{tabular}




\begin{tabular}{|c|c|c|c|c|c|c|c|c|c|c|c|c|c|c|c|c|c|c|c|c|}
\hline ZY1-YX-28 & 2858 & Grey dolomite & 5.87 & 0.25 & 2.82 & 0.06 & 1.09 & 41.02 & 1.22 & 0.12 & 0.06 & 39.31 & 49.43 & 6.74 & 60.32 & 13.3 & 36.98 & 1.474 & 0.76 & 37.24 \\
\hline ZY1-YX-186 & 2863 & Grey dolomite & 10.97 & 0.52 & 4.58 & 0.03 & 1.52 & 18.12 & 0.82 & 0.85 & 0.07 & 100.29 & 102.3 & 9.02 & 88.21 & 14.47 & 81.59 & 3.789 & 4.39 & 49.63 \\
\hline ZY1-YX-193 & 2871 & Gray black mudstone & 11.01 & 0.54 & 4.16 & 0.02 & 1.53 & 18.42 & 0.98 & 1.22 & 0.07 & 110.17 & 172.19 & 9.27 & 57.39 & 20.09 & 80.64 & 3.681 & 3.2 & 53.09 \\
\hline ZY1-8-02 & 2877.4 & Grey black shale & 16.59 & 0.78 & 7.7 & 0.03 & 1.84 & 13.61 & 0.74 & 0.05 & 0.05 & 53.1 & 90.49 & 10.6 & 39.1 & 7.1 & 1.5 & 0 & 3.07 & 4.9 \\
\hline ZY1-8-04 & 2879.4 & Grey black shale & 21.38 & 1 & 5.34 & 0.01 & 2.85 & 6.01 & 1.77 & 0.06 & 0.03 & 51.8 & 85.67 & 9.2 & 32.4 & 4.9 & 1.1 & 0 & 2.63 & 3.2 \\
\hline ZY1-8-06 & 2881.5 & Grey black shale & 18.98 & 0.92 & 4.25 & 0.02 & 2.73 & 2.46 & 1.63 & 0.07 & 0.04 & 44.4 & 77.68 & 8.8 & 31.1 & 5 & 1 & 0 & 2.24 & 3 \\
\hline ZY1-8-08 & 2883.5 & Grey black shale & 16.55 & 0.7 & 5.06 & 0.02 & 2.28 & 14.19 & 0.95 & 0.06 & 0.07 & 46.6 & 79.94 & 9.6 & 36.5 & 7 & 1.4 & 0 & 2.51 & 4.4 \\
\hline ZY1-8-10 & 2885.5 & Grey black shale & 19.86 & 0.84 & 5.08 & 0.02 & 2.7 & 8.96 & 1.08 & 0.06 & 0.12 & 41.8 & 70.3 & 8.5 & 31.5 & 5.7 & 1.3 & 0 & 2.37 & 4 \\
\hline ZY1-9-02 & 2886.5 & Grey black shale & 15.38 & 0.66 & 4.12 & 0.02 & 2.07 & 15.41 & 0.91 & 0.05 & 0.05 & 37.7 & 62.68 & 7.6 & 28 & 4.9 & 1 & 0 & 2.04 & 3.4 \\
\hline ZY1-9-04 & 2888.5 & Grey black shale & 18.12 & 0.83 & 4.69 & 0.02 & 2.6 & 8.33 & 1.05 & 0.05 & 0.06 & 38.6 & 61.77 & 7.3 & 26.1 & 3.9 & 0.9 & 0 & 1.9 & 2.6 \\
\hline ZY1-9-06 & 2890.5 & Grey black shale & 15.46 & 0.76 & 4.6 & 0.02 & 2.09 & 11.48 & 0.88 & 0.05 & 0.11 & 39.3 & 68.5 & 8.1 & 30.4 & 5.5 & 1.2 & 0 & 2.44 & 3.9 \\
\hline ZY1-9-08 & 2892.5 & Grey black shale & 16.46 & 0.79 & 4.79 & 0.02 & 2.26 & 10.95 & 0.97 & 0.05 & 0.07 & 42.3 & 75.07 & 8.9 & 33.2 & 6 & 1.1 & 0 & 2.2 & 3.7 \\
\hline ZY1-9-10 & 2894.4 & Grey black shale & 5.05 & 0.2 & 1.89 & 0.05 & 0.58 & 40.9 & 0.22 & 0.02 & 0.19 & 18.3 & 23.79 & 3.5 & 14.1 & 2.9 & 0.7 & 0 & 1.27 & 2.5 \\
\hline ZY1-YX-32 & 2898 & Gray marl bearing rock & 2.9 & 0.11 & 1 & 0.02 & 0.7 & 51.9 & 0.04 & 0.03 & 0.03 & 22 & 31.61 & 3.58 & 52.24 & 8.89 & 15.58 & 1.385 & 0.46 & 23.87 \\
\hline ZY1-YX-206 & 2903 & Gray black mudstone & 18.1 & 0.83 & 5.46 & 0.02 & 2.58 & 7.75 & 1.64 & 0.31 & 0.13 & 160.46 & 228.14 & 12.99 & 61.67 & 17.25 & 93.1 & 3.98 & 2.88 & 137.53 \\
\hline ZY1-YX-33 & 2914 & Gray marl bearing rock & 3.05 & 0.12 & 1.09 & 0.02 & 0.77 & 52.17 & 0 & 0.02 & 0.03 & 26.31 & 29.44 & 3.41 & 0 & 0 & 17.34 & 1.411 & 1.11 & 24.31 \\
\hline ZY1-YX-220 & 2919 & Dark gray limestone & 1.91 & 0.08 & 0.61 & 0 & 0.19 & 52.95 & 0.1 & 0.18 & 0.06 & 0 & 0 & 0 & 0 & 0 & 0 & 0 & 0 & 12.24 \\
\hline ZY1-YX-225 & 2924 & Dark gray limestone & 2.84 & 0.12 & 0.79 & 0.01 & 0.37 & 48.95 & 0.67 & 0.21 & 0.07 & 0 & 0 & 0 & 0 & 0 & 0 & 0 & 0 & 14.9 \\
\hline ZY1-10-2 & 2927.2 & Dark gray limestone & 12.8 & 0.51 & 3.2 & 0.01 & 2.43 & 5.63 & 0.78 & 0.11 & 0.49 & 153.2 & 174.86 & 8.15 & 86.3 & 29 & 95.66 & 10.684 & 7.11 & 147.05 \\
\hline ZY1-10-7 & 2931.95 & Grey black shale & 17.2 & 0.8 & 4.76 & 0.01 & 3.28 & 9.42 & 0.64 & 0.15 & 0.06 & 92.95 & 174.51 & 12.2 & 44.62 & 14.36 & 58.79 & 3.492 & 0.87 & 193.41 \\
\hline ZY1-YX-36 & 2938 & Grey limestone & 2.94 & 0.11 & 0.97 & 0.02 & 0.71 & 52.33 & 0.17 & 0.03 & 0.03 & 23.39 & 53.31 & 3.15 & 49.87 & 8.88 & 15.71 & 1.571 & 1 & 22.89 \\
\hline ZY1-11-6 & 2944.1 & Dark gray limestone & 1.21 & 0.03 & 0.18 & 0.01 & 0.15 & 57.13 & 0.14 & 0.03 & 0.04 & 0 & 20.37 & 2.02 & 0 & 0.4 & 4.08 & 0.375 & 0 & 6.56 \\
\hline ZY1-12-5 & 2950.1 & Grey black shale & 18.33 & 0.79 & 4.88 & 0.01 & 3.6 & 5.01 & 0.52 & 0.15 & 0.06 & 106.29 & 155.59 & 12.19 & 44.69 & 18.53 & 73.81 & 5.462 & 0.59 & 196.11 \\
\hline ZY1-YX-234 & 2962 & Dark gray argillaceous limestone & 2.16 & 0.09 & 0.6 & 0.01 & 0.27 & 50.75 & 0.25 & 0.56 & 0.04 & 17.23 & 41.2 & 2.79 & 0 & 3.24 & 18.82 & 0.928 & 1.79 & 9.98 \\
\hline ZY1-YX-39 & 2968 & Dark gray argillaceous limestone & 12.52 & 0.58 & 4.35 & 0.02 & 2.01 & 16.34 & 1.28 & 0.28 & 0.08 & 130.16 & 169.42 & 8.75 & 56.49 & 17.19 & 105.75 & 4.444 & 6.41 & 82 \\
\hline ZY1-YX-247 & 2976 & Dark gray argillaceous limestone & 7.42 & 0.33 & 2.17 & 0.01 & 1.21 & 21.97 & 0.53 & 0.12 & 0.08 & 104.95 & 100.79 & 5.42 & 60.86 & 12.86 & 49.39 & 4.454 & 0 & 37.32 \\
\hline
\end{tabular}


Table S2 ZY-1 Well element enrichment coefficient, paleoproduction index and terrigenous input index

\begin{tabular}{|c|c|c|c|c|c|c|c|c|c|c|c|c|c|c|c|c|}
\hline Sample No & Depth & Lithology & $\mathrm{V}_{\mathrm{EF}}$ & $\mathrm{Mo}_{\mathrm{EF}}$ & $\mathrm{U}_{\mathrm{EF}}$ & $\mathrm{Ni}_{\mathrm{EF}}$ & $\mathrm{Cr}_{\mathrm{EF}}$ & $\mathrm{Co}_{\mathrm{EF}}$ & $\mathrm{Cu}_{\mathrm{EF}}$ & $\mathrm{Zn}_{\mathrm{EF}}$ & $\mathrm{Rb} / \mathrm{K}$ & $\mathrm{Fe} / \mathrm{Mn}$ & $\mathrm{Ti} / \mathrm{Al}$ & $\mathrm{Baxs}_{\mathrm{x}}$ & $\mathrm{P} / \mathrm{Al}$ & CIA \\
\hline ZY1-YX-1 & 2668 & Gray black mudstone & 0.698646 & 0.781312 & 0.613037 & 0.935272 & 1.193361 & 0.148765 & 0.418021 & 0.689239 & 43.57911 & 207.6667 & 0.042011 & -0.02747 & 0.005092 & 80.44035 \\
\hline ZY1-YX-3 & 2688 & Dark gray mudstone & 0.645858 & 0.595553 & 0.547912 & 0.795103 & 1.123527 & 0.121585 & 0.360847 & 0.439869 & 40.94375 & 186.6667 & 0.042389 & -0.03651 & 0.003211 & 81.30548 \\
\hline ZY1-4-05 & 2699 & Gray black mudstone & 0.661765 & 0.424909 & 0.487484 & 0.779862 & 0.591983 & 0.129409 & 0.292881 & 0.554408 & 44.76636 & 290.5 & 0.042025 & -0.09325 & 0.002865 & 82.24666 \\
\hline ZY1-YX-5 & 2702 & Gray black mudstone & 0.576939 & 0.470768 & 0.613196 & 0.700534 & 1.035581 & 0.149712 & 0.33774 & 0.551065 & 47.18552 & 208.6667 & 0.049561 & 0.530958 & 0.005646 & 79.89975 \\
\hline ZY1-YX-76 & 2709 & Gray black mudstone & 0.685817 & 0.5248 & 0.588343 & 0.785782 & 1.097871 & 0.133308 & 0.361921 & 0.551186 & 42.87288 & 204.6667 & 0.04 & 0.055417 & 0.006567 & 79.95227 \\
\hline ZY1-YX-85 & 2719 & Gray black mudstone & 0.690821 & 0.288093 & 0.608964 & 1.031254 & 0.877336 & 0.160419 & 0.423607 & 0.48294 & 45.5836 & 196.3333 & 0.040478 & -0.06309 & 0.003981 & 80.24494 \\
\hline ZY1-YX-8 & 2728 & Gray black mudstone & 0.48561 & 0 & 0.424438 & 0.570728 & 0.881579 & 0.103695 & 0.230454 & 0.442625 & 24.55238 & 158 & 0.050771 & 0.503557 & 0.006427 & 79.63153 \\
\hline ZY1-YX-9 & 2738 & Grey black shale & 0.536268 & 0.231945 & 0.516674 & 0.798335 & 0.686684 & 0.13773 & 0.306418 & 0.593459 & 25.16477 & 240.5 & 0.050354 & 0.953053 & 0.014162 & 79.28883 \\
\hline ZY1-YX-11 & 2750 & Gray black mudstone & 0.679757 & 0.412043 & 0.689848 & 0.751804 & 0.756168 & 0.12642 & 0.388286 & 0.658298 & 39.41848 & 278 & 0.041344 & 0.214104 & 0.004522 & 79.62963 \\
\hline ZY1-YX-119 & 2759 & Gray black mudstone & 0.681636 & 0.284703 & 0.592792 & 0.935246 & 0.832911 & 0.181045 & 0.41316 & 1.143063 & 39.05172 & 187 & 0.045326 & 0.833408 & 0.010623 & 78.88268 \\
\hline ZY1-YX-16 & 2770 & Dark gray argillaceous limestone & 1.93518 & 0.44545 & 1.003042 & 1.333858 & 0.841322 & 0.173517 & 0.456987 & 0.747165 & 34.63725 & 92.75 & 0.042234 & 0.019789 & 0.009537 & 85.34884 \\
\hline ZY1-YX-136 & 2787 & Gray black mudstone & 0.649654 & 0.664493 & 0.643714 & 0.606128 & 1.122136 & 0.133038 & 0.340983 & 0.742494 & 51.47396 & 287 & 0.04863 & 0.890124 & 0.014384 & 79.86871 \\
\hline ZY1-6-01 & 2796 & Grey black shale & 0.180355 & 0.648358 & 0 & 0.29278 & 0.399982 & 0.082164 & 0.079435 & 0.00574 & 1.116505 & 505 & 0.049398 & -0.06802 & 0.002533 & 72.3427 \\
\hline ZY1-6-03 & 2798.2 & Grey black shale & 0.222222 & 0.820738 & 0 & 0.386868 & 0.490711 & 0.10707 & 0.113777 & 0.008136 & 1.456693 & 677 & 0.043671 & -0.07375 & 0.002764 & 79.65654 \\
\hline ZY1-6-05 & 2800.5 & Grey black shale & 0.169111 & 0.645782 & 0 & 0.27563 & 0.360672 & 0.077493 & 0.077493 & 0.004909 & 1.09434 & 538 & 0.047229 & -0.09195 & 0.001928 & 83.4115 \\
\hline ZY1-6-07 & 2802.6 & Grey black shale & 0.184468 & 0.868666 & 0 & 0.377299 & 0.456601 & 0.100907 & 0.133197 & 0.009517 & 2.238806 & 625 & 0.053421 & -0.07397 & 0.013205 & 81.22867 \\
\hline ZY1-6-09 & 2804.7 & Grey black shale & 0.191938 & 0.683193 & 0 & 0.331371 & 0.408399 & 0.090893 & 0.101647 & 0.007041 & 1.456693 & 722 & 0.045078 & -0.07203 & 0.004145 & 80.68562 \\
\hline ZY1-7-02 & 2807.55 & Grey black shale & 0.190424 & 0.714478 & 0 & 0.326367 & 0.394719 & 0.089533 & 0.09997 & 0.007157 & 1.607143 & 616 & 0.041623 & -0.06984 & 0.004215 & 82.09343 \\
\hline ZY1-7-04 & 2808.5 & Grey black shale & 0.257305 & 1.174851 & 0 & 0.528799 & 0.588608 & 0.135408 & 0.213229 & 0.015018 & 3.085714 & 471 & 0.061622 & -0.0477 & 0.00936 & 79.3808 \\
\hline ZY1-7-06 & 2810.5 & Grey black shale & 0.175228 & 0.675295 & 0 & 0.315078 & 0.386514 & 0.084862 & 0.098516 & 0.006713 & 1.330935 & 651 & 0.043478 & -0.07846 & 0.002964 & 82.95082 \\
\hline ZY1-7-08 & 2812.5 & Grey black shale & 0.180616 & 0.769044 & 0 & 0.326864 & 0.424591 & 0.08966 & 0.101875 & 0.007036 & 1.675676 & 557 & 0.056522 & -0.07014 & 0.006211 & 84.70712 \\
\hline ZY1-7-10 & 2814.5 & Grey black shale & 0.184656 & 0.755634 & 0 & 0.319749 & 0.378917 & 0.086201 & 0.095262 & 0.006835 & 1.446281 & 626 & 0.046626 & -0.07471 & 0.005485 & 82.05551 \\
\hline ZY1-YX-151 & 2823 & Gray black mudstone & 0.80517 & 0.756206 & 0.668921 & 0.563443 & 1.107853 & 0.106832 & 0.302546 & 0.445735 & 51.58009 & 535 & 0.047404 & 0.028781 & 0.004515 & 77.61717 \\
\hline ZY1-YX-25 & 2828 & Gray black mudstone & 0.688169 & 0 & 0.601484 & 0.808793 & 1.249308 & 0.146946 & 0.326578 & 0.627251 & 30.69048 & 145 & 0.04827 & 0.304888 & 0.007286 & 79.33526 \\
\hline ZY1-YX-160 & 2834 & Gray black mudstone & 0.959659 & 1.95055 & 0.890175 & 0.774731 & 1.258756 & 0.131568 & 0.641465 & 0.999604 & 54.83482 & 506 & 0.047962 & 1.205896 & 0.004197 & 71.64948 \\
\hline ZY1-YX-26 & 2838 & Gray black mudstone & 0.664972 & 0.287612 & 0.640677 & 0.989934 & 0.851489 & 0.170782 & 0.37996 & 0.735892 & 29.13816 & 159.25 & 0.047805 & 0.249882 & 0.00878 & 78.90685 \\
\hline ZY1-YX-172 & 2847 & Grey dolomite & 3.68462 & 2.391493 & 0.802398 & 0.714203 & 1.192816 & 0.113523 & 0.375616 & 0.858403 & 48.44076 & 257.5 & 0.046693 & 0.354515 & 0.006485 & 72.29255 \\
\hline ZY1-YX-28 & 2858 & Grey dolomite & 2.41979 & 0.693969 & 0.721038 & 1.877701 & 0.815702 & 0.209812 & 0.728666 & 0.713388 & 34.16514 & 47 & 0.042589 & 0.079845 & 0.010221 & 62.44681 \\
\hline
\end{tabular}




\begin{tabular}{|c|c|c|c|c|c|c|c|c|c|c|c|c|c|c|c|c|}
\hline ZY1-YX-186 & 2863 & Grey dolomite & 2.58964 & 2.144979 & 0.99178 & 1.469313 & 0.903331 & 0.150247 & 0.424205 & 0.842226 & 32.65132 & 152.6667 & 0.047402 & 0.774956 & 0.006381 & 77.63623 \\
\hline ZY1-YX-193 & 2871 & Gray black mudstone & 0.75188 & 1.557857 & 0.960013 & 0.95247 & 1.51495 & 0.153849 & 0.586825 & 0.829393 & 34.69935 & 208 & 0.049046 & 1.144683 & 0.006358 & 75.93103 \\
\hline ZY1-8-02 & 2877.4 & Grey black shale & 0.240503 & 0.991875 & 0 & 0.430659 & 0.528365 & 0.11675 & 0.137635 & 0.010236 & 2.663043 & 256.6667 & 0.047016 & -0.06349 & 0.003014 & 83.32496 \\
\hline ZY1-8-04 & 2879.4 & Grey black shale & 0.182052 & 0.659346 & 0 & 0.27691 & 0.388148 & 0.078631 & 0.073706 & 0.005824 & 1.122807 & 534 & 0.046773 & -0.08626 & 0.001403 & 76.98956 \\
\hline ZY1-8-06 & 2881.5 & Grey black shale & 0.175776 & 0.632583 & 0 & 0.299409 & 0.396454 & 0.084719 & 0.084719 & 0.005967 & 1.098901 & 212.5 & 0.048472 & -0.05984 & 0.002107 & 76.01121 \\
\hline ZY1-8-08 & 2883.5 & Grey black shale & 0.211573 & 0.812904 & 0 & 0.402992 & 0.46789 & 0.10599 & 0.136022 & 0.00958 & 1.929825 & 253 & 0.042296 & -0.05322 & 0.00423 & 79.83599 \\
\hline ZY1-8-10 & 2885.5 & Grey black shale & 0.15815 & 0.639635 & 0 & 0.289823 & 0.342887 & 0.078207 & 0.0923 & 0.007411 & 1.481481 & 254 & 0.042296 & -0.07586 & 0.006042 & 80.33981 \\
\hline ZY1-9-02 & 2886.5 & Grey black shale & 0.184186 & 0.71095 & 0 & 0.332662 & 0.394777 & 0.090295 & 0.102462 & 0.007364 & 1.642512 & 206 & 0.042913 & -0.05521 & 0.003251 & 79.81318 \\
\hline ZY1-9-04 & 2888.5 & Grey black shale & 0.160067 & 0.562031 & 0 & 0.263199 & 0.330218 & 0.073616 & 0.06922 & 0.005623 & 1 & 234.5 & 0.045806 & -0.07396 & 0.003311 & 79.40403 \\
\hline ZY1-9-06 & 2890.5 & Grey black shale & 0.19101 & 0.84595 & 0 & 0.359307 & 0.429199 & 0.095738 & 0.114412 & 0.008792 & 1.866029 & 230 & 0.049159 & -0.05576 & 0.007115 & 80.06214 \\
\hline ZY1-9-08 & 2892.5 & Grey black shale & 0.1931 & 0.716404 & 0 & 0.368565 & 0.441789 & 0.098801 & 0.117232 & 0.00757 & 1.637168 & 239.5 & 0.047995 & -0.0626 & 0.004253 & 79.67086 \\
\hline ZY1-9-10 & 2894.4 & Grey black shale & 0.27229 & 1.347961 & 0 & 0.510189 & 0.456331 & 0.126643 & 0.184679 & 0.015695 & 4.310345 & 37.8 & 0.039604 & -0.01455 & 0.037624 & 83.19605 \\
\hline ZY1-YX-32 & 2898 & Gray marl bearing rock & 4.898 & 0.850209 & 1.371353 & 3.291613 & 1.055858 & 0.225576 & 0.985871 & 0.608371 & 34.1 & 50 & 0.037931 & 0.010162 & 0.010345 & 78.80435 \\
\hline ZY1-YX-206 & 2903 & Gray black mudstone & 0.666132 & 0.852864 & 0.631399 & 0.622585 & 1.220958 & 0.131139 & 0.306497 & 0.582466 & 53.3062 & 273 & 0.045856 & 0.186182 & 0.007182 & 75.54257 \\
\hline ZY1-YX-33 & 2914 & Gray marl bearing rock & 4.65711 & 1.950688 & 1.328388 & 0 & 0.935008 & 0.204295 & 0 & 0.643793 & 31.57143 & 54.5 & 0.039344 & -0.00086 & 0.009836 & 79.84293 \\
\hline ZY1-YX-220 & 2919 & Dark gray limestone & 0 & 0 & 0 & 0 & 0 & 0 & 0 & 0 & 64.42105 & 0 & 0.041885 & 0.166934 & 0.031414 & 83.04348 \\
\hline ZY1-YX-225 & 2924 & Dark gray limestone & 0 & 0 & 0 & 0 & 0 & 0 & 0 & 0 & 40.27027 & 79 & 0.042254 & 0.190572 & 0.024648 & 62.41758 \\
\hline ZY1-10-2 & 2927.2 & Dark gray limestone & 3.32911 & 2.977313 & 2.396746 & 1.231983 & 1.323299 & 0.116348 & 0.728623 & 0.846289 & 60.5144 & 320 & 0.039844 & 0.022438 & 0.038281 & 76.23585 \\
\hline ZY1-10-7 & 2931.95 & Grey black shale & 0.406063 & 0.271118 & 0.582969 & 0.474026 & 0.982813 & 0.12961 & 0.268499 & 0.387053 & 58.96646 & 476 & 0.046512 & 0.032338 & 0.003488 & 79.04412 \\
\hline ZY1-YX-36 & 2938 & Grey limestone & 4.83136 & 1.82313 & 1.534359 & 3.099527 & 1.756465 & 0.195778 & 0.971366 & 0.605097 & 32.23944 & 48.5 & 0.037415 & 0.009888 & 0.010204 & 73.68421 \\
\hline ZY1-11-6 & 2944.1 & Dark gray limestone & 0 & 0 & 0.889905 & 0 & 1.630738 & 0.305048 & 0.106313 & 0.381832 & 43.73333 & 18 & 0.024793 & 0.021723 & 0.033058 & 73.78049 \\
\hline ZY1-12-5 & 2950.1 & Grey black shale & 0.435715 & 0.172528 & 0.855631 & 0.445503 & 0.822235 & 0.121517 & 0.325108 & 0.455987 & 54.475 & 488 & 0.043099 & 0.024608 & 0.003273 & 79.79974 \\
\hline ZY1-YX-234 & 2962 & Dark gray argillaceous limestone & 6.57602 & 4.441854 & 1.233649 & 0 & 1.847659 & 0.236024 & 0.4824 & 0.986648 & 36.96296 & 60 & 0.041667 & 0.545224 & 0.018519 & 73.72014 \\
\hline ZY1-YX-39 & 2968 & Dark gray argillaceous limestone & 3.40356 & 2.744219 & 1.019219 & 0.824462 & 1.310804 & 0.127706 & 0.441556 & 0.956474 & 40.79602 & 217.5 & 0.046326 & 0.194353 & 0.00639 & 73.25922 \\
\hline ZY1-YX-247 & 2976 & Dark gray argillaceous limestone & 5.74294 & 0 & 1.72363 & 1.498758 & 1.315803 & 0.133477 & 0.557381 & 0.753762 & 30.84298 & 217 & 0.044474 & 0.069241 & 0.010782 & 76.57379 \\
\hline
\end{tabular}


